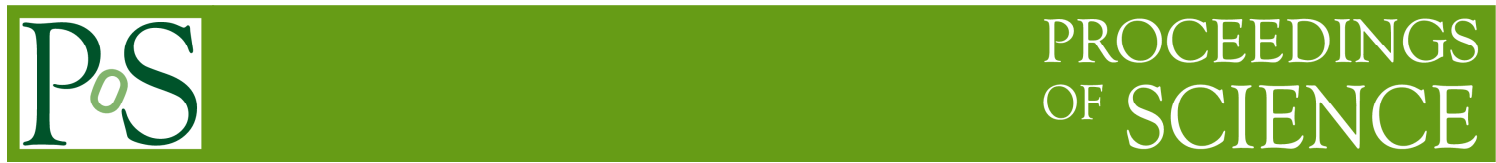

\title{
Results from the NA48/2 experiment: Tests of chiral perturbation theory with radiative kaon decays
}

\section{Evelina Marinova*i}

University of Sofia 'St. Kliment Ohridski'

E-mail: Evelina.Marinova@cern.ch

The NA48/2 experiment at the CERN SPS whose main goal was to study direct CP violation in the system of charged kaons collected enough statistics to allow the study of rare decay modes and to check the predictions of Chiral Perturbation Theory for their parameters and decay rates. The experiment took data in 2003 and 2004, collecting samples of 1,164 $K^{ \pm} \rightarrow \pi^{ \pm} \gamma \gamma$ candidates with $3.3 \%$ background, $120 K^{ \pm} \rightarrow \pi^{ \pm} e^{+} e^{-} \gamma$ candidates with $6.1 \%$ background and $7,146 K^{ \pm} \rightarrow$ $\pi^{ \pm} e^{+} e^{-}$decay candidates with $0.6 \%$ background. The latest results on precise measurements of the branching ratios and of the form factor parameters of these decay modes are presented.

8th Conference Quark Confinement and the Hadron Spectrum

September 1-62008

Mainz, Germany

\footnotetext{
${ }^{*}$ Speaker.

On behalf of NA48/2 Collaboration
} 


\section{Introduction}

The experiment NA48/2 at the CERN SPS took data in 200)3 and 20044 with the aim of collecting the highest existing statistics of charged kaon decays. The main goal of the experiment was to search for direct CP violation in this system where it was not yet observed, and to perform the most precise measurements of other parameters of the Standard Model. Given the high statistics achieved, also semileptonic, leptonic and rare charged kaon decays were studied. Rare kaon decays offer the possibility of probing high energy scales by doing precise low energy measurements. In particular, radiative kaon decays are sensitive to the non perturbative aspects of the SM. The SM in the hadronic sector at low energies is described by the Chiral Perturbation Theory (ChPT) which is a non-decoupling effective field theory. In the following sections, recent results on ChPT parameters obtained by the NA48/2 Collaboration will be presented.

\section{Chiral perturbation theory}

Since at low energies $(E \ll 1 G e V)$ QCD cannot be treated perturbatively, an effective theory has to be used - ChPT. The relevant degrees of freedom of this model are not quarks and gluons, but the light pseudo-scalar mesons $\eta, \pi$ and $K$ (the pseudo-Goldstone bosons of spontaneously broken chiral symmetry). ChPT contains, as every effective theory, free parameters, whose values have to be determined experimentally. Complete descriptions of the theory can be found for example in [1], [2], [3].

\section{The NA48/2 experiment}

The NA48/2 apparatus and beam line are upgraded and modified versions of the previous NA48 experiments [4]. The improved set-up includes a new beam line, two new detectors (beam spectrometer and beam monitor), and an extended decay region. The primary protons produced by the SPS accelerator have a nominal momentum of $400 \mathrm{GeV} / \mathrm{c}$. The beam line had been designed to simultaneously deliver positive and negative beams of average momentum $60 \mathrm{GeV} / \mathrm{c}$, produced at a Be target. The beam focusing has been realized with a system of magnets of alternate polarity and the beams are steered in such a way to coincide at the end of the decay region.

The most important subdetectors used in these analyses are the magnetic spectrometer which is composed of four high resolution drift chambers $(\mathrm{DCH})$ and a dipole magnet located after the first two chambers, the charged hodoscope (CHOD) which is used to provide a fast topological trigger for events with charged particles and to measure their time, and the electromagnetic calorimeter (LKr) which is a liquid Krypton quasi-homogenous ionization chamber.

\section{Experimental results}

\subsection{The $K^{ \pm} \rightarrow \pi^{ \pm} \gamma \gamma$ decay}

The $K^{ \pm} \rightarrow \pi^{ \pm} \gamma \gamma$ decay has no tree level diagram with $O\left(p^{2}\right)$ and the leading contribution appears at $O\left(p^{4}\right)$. The $O\left(p^{6}\right)$ unitarity corrections can increase the BR by 30-40\% [5]. Both decay spectrum and rate (see Fig. 1(left)) depend strongly on the single parameter $\hat{c}$. 
Results from the NA48/2 experiment: Tests of chiral perturbation theory with radiative kaon decays Evelina Marinova
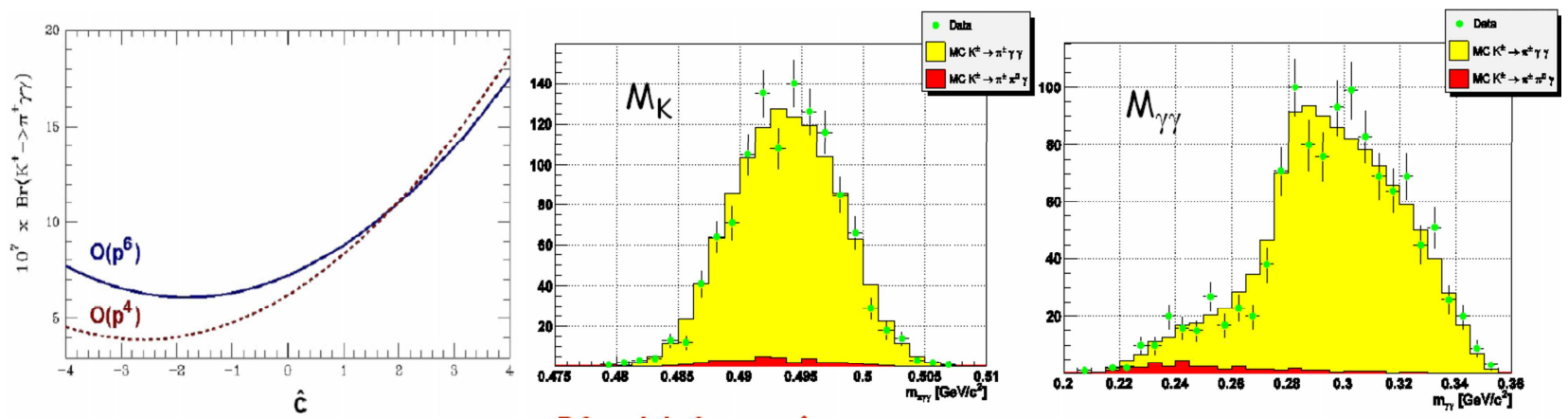

Figure 1: Theoretical predictions for the Branching ratio of $K^{ \pm} \rightarrow \pi^{ \pm} \gamma \gamma$ at $O\left(p^{4}\right)$ and $O\left(p^{6}\right)$ with respect to the value of the parameter $\hat{c}$ [5] (left). The reconstructed invariant mass of the kaon (middle). The invarinat mass $M_{\gamma \gamma}$ (right).

NA48/2 has analysed about $40 \%$ of its data, finding 1164 signal candidates with $3.3 \%$ background. That is 40 times larger statistics than the one collected from the previous experiment E787 at BNL $[6]$ ( $31 \pm 5$ events). The reconstructed $M_{\gamma \gamma}$ in the accessible kinematical region $M_{\gamma \gamma}>0.2$ and $M_{\pi \gamma \gamma}$ spectra can be seen in Fig. 1 (right and middle) for selected candidates (crosses), signal MC (yellow) and background (red). The signal and the normalization channels have identical particle composition of the final states, and the only cut to distinguish between the two channels is the $M_{\gamma \gamma}$ invariant mass cut.

The rate of $K^{ \pm} \rightarrow \pi^{ \pm} \gamma \gamma$ has been measured with respect to the normalization channel $K^{ \pm} \rightarrow$ $\pi^{ \pm} \pi^{0}$. The preliminary result for the model dependent branching fraction (assuming $O\left(p^{6}\right)$ prediction $\lceil 5\rceil$ and using $\hat{c}=2$., based on the measurement of [6] $)$ is $B R=\left(1.07 \pm 0.04_{\text {stat }} \pm 0.08_{\text {syst }}\right) \times$ $10^{-6}$. A model independent $B R$ measurement is in preparation, together with the extraction of $\hat{c}$ from a fit to $M_{\gamma \gamma}$.

\subsection{The $K^{ \pm} \rightarrow \pi^{ \pm} e^{+} e^{-} \gamma$ decay}
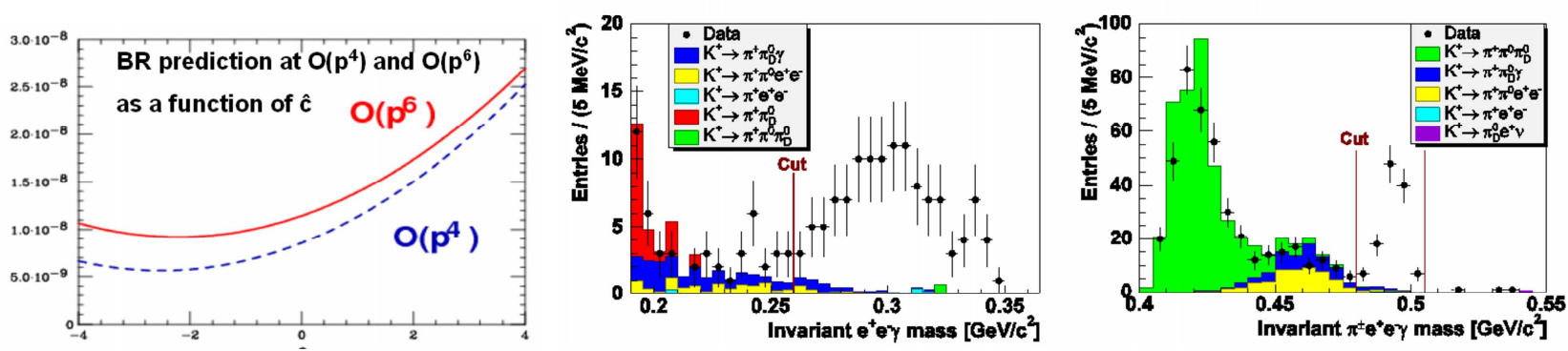

Figure 2: Theoretical predictions for the Branching ratio of $K^{ \pm} \rightarrow \pi^{ \pm} e^{+} e^{-} \gamma$ at $O\left(p^{4}\right)$ and $O\left(p^{6}\right)$ with respect to the value of the parameter $\hat{c}$ [7] (left). A cut, rejecting the background, is applied on the invariant mass $M_{e e \gamma}>0.26$ (middle). The reconstructed invariant mass of the kaon (right).

The theory for this decay is similar to the theory for $K^{ \pm} \rightarrow \pi^{ \pm} \gamma \gamma$ with one photon internally converting into a pair of electrons. The naive expectation for the branching ratio of this decay is $B R\left(K^{ \pm} \rightarrow \pi^{ \pm} e^{+} e^{-} \gamma\right) \approx \alpha \cdot B R\left(K^{ \pm} \rightarrow \pi^{ \pm} \gamma \gamma\right)$. The theoretical prediction of $|7|$ is in the range $\sim(0.9-1.6) \times 10^{-8}$ depending on the value of the parameter $\hat{c}$ (see Fig. 2 (left)).

NA48/2 has reported the first observation of the decay $K^{ \pm} \rightarrow \pi^{ \pm} e^{+} e^{-} \gamma$ using the full 2003 and 2004 data sample [8]. 120 events were found in the signal region with an estimated background of 
$7.3 \pm 1.7$ events. The signal events and the estimated background identified with the help of MC simulation can be seen in Fig. 2 (middle and right). The particles in the final state are the same.

A cut is applied on the invariant mass $M_{e e \gamma}>0.26$ in order to suppress the background. Using $K \rightarrow \pi^{ \pm} \pi_{D}^{0}$ as a normalization channel, the branching ratio has been measured (in a model independent way) to be $B R_{m i}=\left(1.19 \pm 0.12_{\text {stat }} \pm 0.04_{\text {syst }}\right) \times 10^{-8}$ for $M_{e e \gamma}>0.26$. The parameter $\hat{c}$ has been measured under the assumption of $O\left(p^{6}\right)|7|$ validity and it is found to be $\hat{c}=0.90 \pm 0.45$. Using this value, model dependent branching ratio is calculated: $B R_{m i}=\left(1.29 \pm 0.13_{\text {exp }} \pm 0.03_{\hat{c}}\right) \times$ $10^{-8}$.

\subsection{The $K^{ \pm} \rightarrow \pi^{ \pm} e^{+} e^{-}$decay}
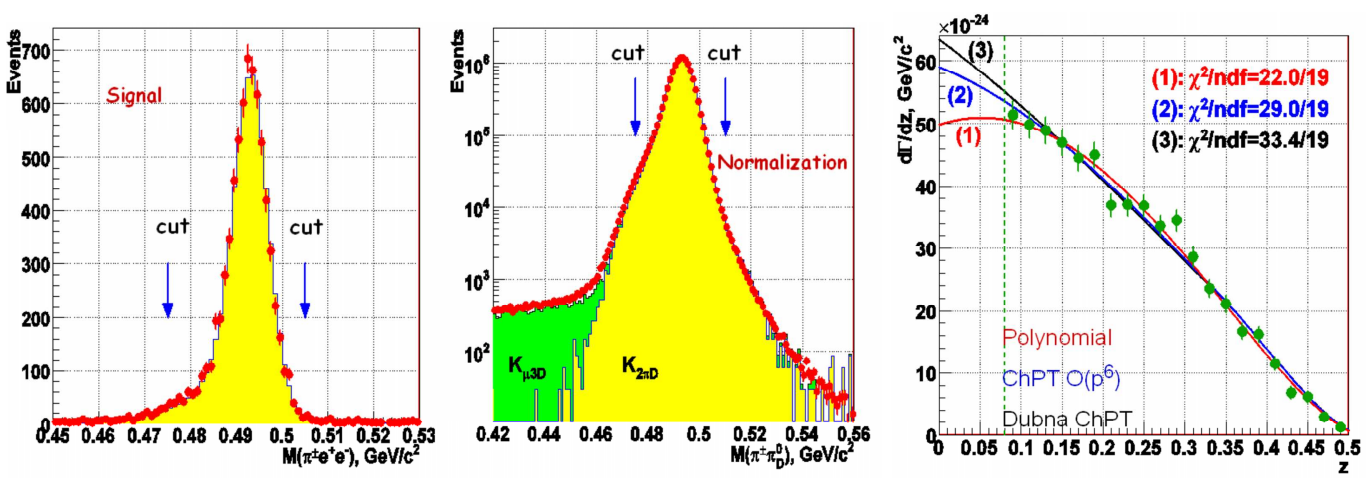

Figure 3: Signal region in terms of the reconstructed kaon mass for $K^{ \pm} \rightarrow \pi^{ \pm} e^{+} e^{-}$events (left) and $K^{ \pm} \rightarrow$ $\pi^{ \pm} \pi_{D}^{0}$ events (middle). Differential decay rate of the $K^{ \pm} \rightarrow \pi^{ \pm} e^{+} e^{-}$decay and fit to the three considered models (right).

The FCNC process $K^{ \pm} \rightarrow \pi^{ \pm} e^{+} e^{-}$, proceeding through a virtual gamma exchange, is described by ChPT in three different models, predicting the form factors characterizing the normalized dilepton invariant mass spectrum, $z=M_{e e}^{2} / M_{K}^{2}$, and the amplitudes of the decay rate:

- Linear: $W(z)=G_{F} M_{K}^{2} f_{0}(1+\lambda z)$ with normalization $f_{0}$ and slope $\lambda$;

- ChPT at $O\left(p^{6}\right): W_{+}(z)=G_{F} M_{K}^{2}\left(a_{+}+b_{+} z\right)+W_{+}^{\pi \pi}(z)$, [9], where $W_{+}^{\pi \pi}(z)$ denotes the contribution from the two-pion intermediate state and $a_{+}, b_{+}$are free parameters;

- Dubna version of ChPT: involving vector meson resonance contribution:

$W(z) \equiv W\left(M_{a}, M_{\rho}, z\right)\lceil 10\rceil$, with resonance masses $\left(M_{a}, M_{\rho}\right)$ treated as free parameters.

This analysis is based on the full data sample and the goal is the extraction of the form factor parameters in the framework of each of the aforementioned models, and computation of the corresponding branching fractions and a measurement of a model independent branching faction in the accessible kinematical region $z>0 .(08$. This cut is applied in order to suppress the background coming from the normalization channel $K^{ \pm} \rightarrow \pi^{ \pm} \pi_{D}^{0}$. The usage of a similar channel, with the same number of charged particles, the same number of leptons, cancels some of the possible systematics arising from particle identification, trigger efficiencies, etc.

In total 7,146 $K^{ \pm} \rightarrow \pi^{ \pm} e^{+} e^{-}$candidates have been found in the signal region with an estimated background of $0.6 \%$ (see Fig. 3(left)). The $K^{ \pm} \rightarrow \pi^{ \pm} \pi_{D}^{0}$ candidates are shown in the middle 
and the three fits to the models are presented on the right. The results are summarized in Tab. 1. The results for tha parameters $\lambda, f_{0}, a_{+}, b_{+}$and for the $B R$ are in agreement with the previous measurements $[11]$. The third model has never been tested before.

The preliminary average result for $B R$ in the full kinematic range is: $B R=\left(3.08 \pm 0.04_{\text {stat }} \pm\right.$ $\left.0.08_{\text {ext }} \pm 0.07_{\text {model }}\right) \times 10^{-7}$. A first measurement of the direct CP violating asymmetry of $K^{+}$and $K^{-}$decay rates in the full kinematic range was obtained: $\Delta\left(K_{\pi e e}^{ \pm}\right)=\left(-2.1 \pm 1.5_{\text {stat }} \pm 0.3_{\text {syst }}\right) \%$. The result is compatible with no $\mathrm{CP}$ violation. However its precision is far from the theoretical expectation [12] of $\left|\Delta\left(K_{\pi e e}^{ \pm}\right)\right| \sim 10^{-4}$.

\begin{tabular}{|c|c|c|c|}
\hline \hline Model & Parameter & Results & Combined error \\
\hline Linear & $\lambda$ & $2.35 \pm 0.15_{\text {stat }} \pm 0.09_{\text {syst }}$ & $2.35 \pm 0.18$ \\
Linear & $f_{0}$ & $0.532 \pm 0.012_{\text {stat }} \pm 0.008_{\text {syst }} \pm 0.007_{\text {ext }}$ & $0.532 \pm 0.016$ \\
Linear & $B R_{1} \times 10^{-7}$ & $3.02 \pm 0.04_{\text {stat }} \pm 0.04_{\text {syst }} \pm 0.08_{\text {ext }}$ & $3.02 \pm 0.10$ \\
\hline \hline ChPT & $a_{+}$ & $-0.579 \pm 0.012_{\text {stat }} \pm 0.008_{\text {syst }} \pm 0.007_{\text {ext }}$ & $-0.579 \pm 0.016$ \\
ChPT & $b_{+}$ & $-0.798 \pm 0.053_{\text {stat }} \pm 0.037_{\text {syst }} \pm 0.017_{\text {ext }}$ & $-0.798 \pm 0.067$ \\
ChPT & $B R_{2} \times 10^{-7}$ & $3.11 \pm 0.04_{\text {stat }} \pm 0.04_{\text {syst }} \pm 0.08_{\text {ext }}$ & $3.11 \pm 0.10$ \\
\hline \hline ChPT(Dubna par.) & $M_{a}$ & $0.965 \pm 0.028_{\text {stat }} \pm 0.018_{\text {syst }} \pm 0.002_{\text {ext }}$ & $0.965 \pm 0.033$ \\
ChPT(Dubna par.) & $M_{\rho}$ & $0.711 \pm 0.010_{\text {stat }} \pm 0.007_{\text {syst }} \pm 0.002_{\text {ext }}$ & $0.711 \pm 0.013$ \\
ChPT(Dubna par.) & $B R_{3} \times 10^{-7}$ & $3.15 \pm 0.04_{\text {stat }} \pm 0.04_{\text {syst }} \pm 0.08_{\text {ext }}$ & $3.15 \pm 0.10$ \\
\hline n/a & $B R_{m i} \times 10^{-7}$ & $2.26 \pm 0.03_{\text {stat }} \pm 0.03_{\text {syst }} \pm 0.06_{\text {ext }}$ & $2.26 \pm 0.08$ \\
\hline \hline
\end{tabular}

Table 1: Results of fits to the three models, and the model-independent branching ratio $B R_{m i}(z>0.08)$.

\section{References}

[1] A. Pich, Lectures at the 1997 Les Houches Summer School, [arXiv:hep-ph/9806303], (1998).

[2] G. Colangelo and G. Isidori, Lectures given at at the 2000 LNF Spring School, Frascati, [arXiv:hep-ph/0101264],(2000).

[3] S. Scherer and M. R. Schindler, Lectures given at the ECT* Doctoral Training Programme 2005 (Marie Curie Training Site) "Hadronic Physics", [arXiv:hep-ph/0505265],(2005).

[4] V. Fanti et al., Nucl. Instrum. Methods A 574, 433 (2007).

「5] G. D'Ambrosio and J. Portoles, Phys. Lett. B 386, 403 (1996).

「6] P. Kitching et al., Phys. Rev. Lett. 79, 4079 (1997).

|7| F. Gabbiani, Phys. Rev. D 59, 094022 (1999).

[8] J.R.Batley, et al., NA48/2 Collaboration. Phys. Lett. B659 (2008) 493.

[9] G. D’Ambrosio et al., JHEP 8 (1998) 4.

[10] A.Z. Dubnickova et al., Phys. Part. Nucl. Lett. 5, vol. 2 (2008) 76; [arxiv:hep-ph/0611175].

[11] P. Bloch et al.(Geneva - Saclay coll.), Phys. Lett. B56 (1975) 201; C. Alliegro et al., Phys. Rev. Lett. 68 (1992) 278.; R. Appel et al. (E865 coll), Phys. Rev. Lett. 83 (1999) 4482.

$\lceil 12\rceil$ G. Ecker, A. Pich and E. de Rafael, Nucl. Phys. B303 (1988) 665. 